\title{
Plastic Section and Cryo-Electron Tomography Using the IMOD Software Package
}

D. Nicastro

Brandeis University, Rosenstiel Center MS029, 415 South Str., Waltham, MA 02453

Electron tomography (ET) is a powerful analytical method for studying complex biological and material sciences specimen in three-dimensions (3D). In ET a set of projection images from different viewing angles is recorded using a transmission electron microscope. These images can then be used to reconstruct the 3D structure of the specimen [1]. In principle a tilt series can be obtained of most electron microscopic samples, but in the life sciences, ET of samples prepared by rapid freezing has been particularly successful due to the outstanding structure preservation and good time resolution of dynamic cellular processes [2]. After plunge-freezing or high-pressure freezing (HPF) the specimen, they are either freeze-substituted (HPF-FS), plastic embedded and sectioned, or imaged directly in the frozen-hydrated state (cryo-ET) [3].

In this technical presentation we give an introduction into the fundamentals of ET, show a few exemplary applications, discuss strengths and limitations of different approaches, and provide a broad overview of the image acquisition software SerialEM [4], the reconstruction and modeling software package IMOD [5] and the Particle Estimation for Electron Tomography (PEET) software for single particle averaging of tomographic volumes [6]. Special emphasis will be on introducing the steps involved in building a tomographic reconstruction with the IMOD software package, including the differences between reconstructing HPF-FS plastic section and cryo-tomograms. The major advantage of cellular ET is that - at its best - it will provide 3D information of a wellpreserved, pleomorphic biological specimen, in some cases with molecular resolution and much potential for the characterization of biological structures and macromolecular assemblies in situ [6]. Such information can provide detailed insights into the structural basis and ultimately the function of many cellular processes.

References

[1] Frank J. (1992) Electron tomography: Three-dimensional imaging with the transmission electron microscope. Plenum Press, N.Y.

[2] Dubochet J. et al. (1988) Quart. Rev. Biophys. 21:129.

[3] McIntosh J.R. et al. (2005) Trends Cell Biol. 15:43.

[4] Mastronarde D. (2005) J Struct Biol. 152:36.

[5] Kremer et al. (1996) J Struct Biol. 116:71.

[6] Nicastro D. et al. (2006) Science 313:944. 